\title{
THE RECURSIVE EQUIVALENCE TYPE OF A DECOMPOSITION OF AN $\omega$-GROUP: THE RET OF A DECOMPOSITION
}

\author{
C. H. APPLEBAUM ${ }^{1}$ \\ (Received 12 October 1970; revised 23 November 1970) \\ Communicated by G. N. Crossley
}

\section{Introduction}

Let $\varepsilon, \Lambda, \Lambda^{*}, \Lambda_{R}$ stand for the set of non-negative integers, isols, isolic integers and regressive isols respectively, and let $P(\tau)$ be the $\omega$-group of Gödel numbers of permutations of the set $\tau \subset \varepsilon$ which move only finitely many elements of $\tau$. The concept of an $\omega$-group was studied by Hassett [5]. He proved in P12 of [5] that for an isolated set $\tau$, the decomposition of $P(\tau)$ into conjugacy sets is a $g c$-decomposition if and only if $\tau$ is regressive. For the finite symmetric group on $n$ elements, $S_{n}$, it is known that the order of the conjugacy class is $p(n)$, where $p(n)$ is the partition function. The author shows in this paper, using a result of Barback [1], that if $p_{\Lambda}(T)$ is Nerode's canonical extension of $p(n)$ to $\Lambda$ and Req $(\tau)=T$, then $p_{\Lambda}(T)=\operatorname{Req} C_{t}$, where $C_{\tau}$ is the decomposition of $P(\tau)$ into conjugacy sets. The reader is assumed to be familiar with the contents of $[2],[4]$ and [5].

\section{Basic concepts}

Notations. (i) A function of $n$, say $a(n)$, may also be written $a_{n}$.

(ii) $v(n)=\{0,1,2, \cdots, n-1\}$.

(iii) In this paper we will denote the group of all finite permutations of a set $\sigma$, i.e. those permutations which move only finitely many elements of $\sigma$, by $\mathscr{P}(\sigma)$. We denote the set of elements in $P(\sigma)$ by $\sigma^{*}$, that is,

$$
\sigma^{*}=\left\{f^{*} \in \varepsilon \mid f \in \mathscr{P}(\sigma)\right\} .
$$

(iv) For a recursive function $f(x)$, we denote Nerode's canonical extension from $\Lambda$ into $\Lambda^{*}$ by $f_{\Lambda}$. We know by [1] that $f_{\Lambda}$ maps $\Lambda_{R}$ into itself if and only if $f$ is eventually increasing.

1 The results presented in this paper were taken from the author's doctoral dissertation written at Rutgers University under the direction of Professor J. C. E. Dekker. 
We need the following theorems, [3, Prop. 1] and [1, p. 36] respectively,

(1) The function $f$ has a one-to-one partial recursive extension if and only if $f$ and $f^{-1}$ have partial recursive extensions and $f$ is one-to-one.

(2) Let $f(n)$ be an increasing recursive function and define $e(n)$ by $e(0)$ $=f(0)$ and $e(n+1)=f(n+1) \neq f(n)$. Then $f_{\Lambda}(T)=\Sigma_{T+1} e_{n}$, for $T \in \Lambda_{R}$.

Definition. A partition of the positive integer $n$ is an expression of the form $n_{1}+\cdots+n_{k}$, where $n_{1}, \cdots, n_{k}$ denote positive integers (not necessarily distinct) with $n$ as sum. Two partitions of $n$ are equal, if they only differ in the order of their terms; we may therefore assume that $1 \leqq n_{1} \leqq n_{2} \leqq \cdots \leqq n_{k} \leqq n$.

Definition. The partition function is the function $p(n)$ from $\varepsilon$ into $\varepsilon$ such that $p(0)=1$ and for $n \geqq 1, p(n)$ is the number of distinct partitions of $n$.

Several properties of $p(n)$ are discussed in Chapter 10 of [7]. It is readily seen that $p(n)$ is an increasing recursive function which is strictly increasing for $n \geqq 1$. Hence $p_{\Lambda}$ is a function from $\Lambda_{R}$ into itself. It is well-known from group theory that for $n \geqq 1, p(n)$ is the number of conjugacy sets of the symmetric group $S_{n}$. Assume that $\tau$ is a non-empty finite set of cardinality $n$. Then $P(\tau) \cong S_{n}$, hence

$$
p(n)=\text { the number of conjugacy sets of } P(\tau) .
$$

Let $\tau$ be a non-empty isolated set and $C_{t}$ the decomposition of the isolic group $P(\tau)$ into conjugacy sets. By [5,P12] the $m d$-class $C_{\tau}$ is a $g c$-class if and only if $\tau$ is regressive. Obviously, if $\tau$ is regressive, $\operatorname{RET}\left(C_{\tau}\right)$ only depends on $\operatorname{Req}(\tau)$.

Notation. If $T \in \Lambda_{R}$ and $T \neq 0, C_{T}=\operatorname{Req}\left(C_{\tau}\right)$, for $\tau \in T$.

We observed that $C_{T}=p(n)$, in case $T=n>0$. It is therefore a reasonable conjecture that $C_{T}=p_{\Lambda}(T)$, for $T \in \Lambda_{R}, T \neq 0$.

\section{Main Result}

THEOREM. If $T$ is a non-zero regressive isol, $C_{T}=p_{\Lambda}(T)$.

Proof. The result holds if $T$ is finite, hence we assume that $T \in \Lambda_{R}-\varepsilon$ Let $\sigma \in T+1, t_{n}$ a regressive function ranging over $\sigma$ and $\tau=\sigma-\left(t_{o}\right)$. Then $\tau \in T$. For $x \in P(\tau)$ we denote the conjugacy set of $x$ by $C(x)$. In the proof of $[5, \mathrm{P} 12]$ a function $h^{*}(x)$ from $\tau^{*}$ into itself is defined as follows: $h^{*}(1)=1$; given any number $x=f^{*} \in P(\tau)$ with $x \neq 1$, that is, $f \neq i$, we can compute the cycle structure of $f$, say

$$
(n(1), \cdots, n(k)) \text {, where } 2 \leqq n(1) \leqq \cdots \leqq n(k) .
$$

Let $n=n(1)+\cdots+n(k)$. Taking into account that $\tau=\left(t_{1}, t_{2}, \cdots\right)$, we define 


$$
h_{f}=\left(t_{1}, \cdots, t_{n(1)}\right)\left(t_{n(1)+1}, \cdots, t_{n(1)+n(2)}\right) \cdots\left(t_{n-n(k)+1}, \cdots, t_{n}\right), h^{*}(x)=\left(h_{f}\right)^{*} .
$$

According to the proof, the function $h^{*}(x)$ is a $g c$-function of the $m d$-class $C_{\tau}=\left\{C(x) \mid x \in \tau^{*}\right\}$. We conclude that

$$
C_{T}=R E T\left(C_{\tau}\right)=\operatorname{Req} h^{*}\left(\tau^{*}\right) .
$$

Let $e(0)=p(0), e(n+1)=p(n+1)-p(n)$. Thus by (2) we have that $p_{\Lambda}(T)$ $=\Sigma_{T+1} e_{n}$, that is,

$$
p_{\Lambda}(T)=\operatorname{Req} \bigcup_{n=0}^{\infty} j\left[t_{n}, v\left(e_{n}\right)\right] .
$$

We define $\beta=\bigcup_{n=0}^{\infty} j\left[t_{n}, v\left(e_{n}\right)\right], \gamma=h^{*}\left(\tau^{*}\right)$.

In view of (3) and (4) the proof will be complete if we can show that $\beta \simeq \gamma$. Let us call a partition $\left(n_{1}, \cdots, n_{k}\right)$ of $n$,

$$
\begin{aligned}
& \text { of the first type, if } 1=n_{1} \leqq n_{2} \leqq \cdots \leqq n_{k} \\
& \text { of the second type, if } 2 \leqq n_{1} \leqq n_{2} \leqq \ldots \leqq n_{k}
\end{aligned}
$$

Let for $n \geqq 1$,

$$
\begin{aligned}
& p_{1}(n)=\text { the number of partitions of } n \text { of the first type, } \\
& p_{2}(n)=\text { the number of partitions of } n \text { of the second type, }
\end{aligned}
$$

then $p(n)=p_{1}(n)+p_{2}(n)$, for $n \geqq 1$. We claim

$$
p_{2}(n)=e(n), \quad \text { for } n \geqq 1 \text {. }
$$

This is trivial for $n=1$, since $p_{2}(1)=0, e(1)=0$. Now consider the numbers $\geqq 2$, that is, the numbers of the form $n+1$, with $n \geqq 1$. If $\left(1, n_{2}, \cdots, n_{k}\right)$ is a first type partition of $n+1$, then $\left(n_{2}, \cdots, n_{k}\right)$ is a partition of $n$. Moreover, all partitions of $n$ can be obtained by dropping the initial " $1+$ " from a first type partition of $n+1$. Hence $p_{1}(n+1)=p(n)$ and

$$
e(n+1)=p(n+1)-p(n)=p(n+1)-p_{1}(n+1)=p_{2}(n+1) .
$$

For $n \geqq 2$ we have $e_{n}>0$, that is, $n$ has second type partitions. If $\left(n_{1}, \cdots, n_{k}\right)$ is a second type partition we denote it by $\left[n_{1}, \cdots, n_{k}\right]$ and define its Gödel number (G-number) by

$$
\left[n_{1}, \cdots, n_{k}\right]^{\#}=p_{1}^{n_{1}} \cdots p_{k}^{n_{k}}
$$

where $p_{i}$ is the $i$ th odd prime. For each $n \geqq 2$ we denote the class of second type partitions of $n$ by $T_{n}$ and order this class according to increasing $G$-numbers, say

$$
T_{n}=\left(s_{n, 0}, s_{n, 1}, \cdots, s_{n, e(n)-1}\right) \text {. }
$$


We define the function $b_{n}(i)$ for $n, i \in \varepsilon$ by

$$
\begin{array}{ll}
b_{n}(i)=\left(s_{n, i}\right)^{\#}, & \text { for } n \geqq 2, i<e_{n}, \\
b_{n}(i)=0, & \text { otherwise. }
\end{array}
$$

Then $b_{n}(i)$ is a recursive function. Let $q(x)$ be a regressing function of $t_{n}$ and

$$
q^{*}(x)=(\mu y)\left[q^{y+1}(x)=q^{y}(x)\right], \text { for } x \in \delta q
$$

Suppose a number

$$
x=j\left(t_{n}, i\right) \in \beta=\bigcup_{n=0}^{\infty} j\left[t_{n}, v\left(e_{n}\right)\right]
$$

is given. Then the numbers

$$
t_{n}=k(x), n=q^{*} k(x), i=l(x),
$$

can be computed. Clearly $i<e_{n}$, because $x \in \beta$. Since $e(0)=p(0)=1, e(1)=0$, $e(m) \geqq 1$, for $m \geqq 2$, we see that the number $n=q^{*} k(x)$ must be 0 or $\geqq 2$. If $n=0$, we have $x=j\left(t_{0}, 0\right)$ and define $g(x)=1$. Now assume $n \geqq 2$. Then the number $b_{n}(i)$ is of the form

$$
b_{n}(i)=\left[n_{1}, \cdots, n_{k}\right]^{\#}, \text { where }\left[n_{1}, \cdots, n_{k}\right] \in T_{n} .
$$

In this case we define

$$
g(x)=\left(\left(t_{1}, \cdots, t_{n(1)}\right) \cdots\left(t_{n-n(k)+1}, \cdots, t_{n}\right)\right)^{*} .
$$

Since the function $t_{n}$ is regressive and the numbers $t_{n}, n, i$ can be computed from $x$, so can the number $g(x)$. We claim

$$
g \text { is one-to-one on } \beta \text {, }
$$

$$
g(\beta)=\gamma
$$

$\operatorname{Re}(6)$. The function $g$ maps $j\left(t_{0}, 0\right)$ onto 1 and any number $x \in \beta$ which is different from $j\left(t_{0}: 0\right)$ onto a member of $\gamma$ which is different from 1 .

Now assume that

$$
x=j\left(t_{n}, i\right) \in \beta, x^{\prime}=j\left(t_{m}, i^{\prime}\right) \in \beta,
$$

where $n, m \geqq 2$ and $x \neq x^{\prime}$. It follows that $n \neq m$, or $n=m$ and $i \neq i^{\prime}$. If $n \neq m$, the classes $T_{n}$ and $T_{m}$ are disjoint, and hence $g(x)$ and $g\left(x^{\prime}\right)$ are $G$-numbers of finite permutations in $\mathscr{P}(\tau)$ whose cycle classes are distinct. On the other hand, if $n=m, i \neq i^{\prime}$, we see that $T_{n}=T_{m}$ and $g(x)$ and $g\left(x^{\prime}\right)$ are $G$-numbers of finite permutations in $\mathscr{P}(\tau)$ whose cycle structures are distinct members of $T_{n}$. In each case $g(x) \neq g\left(x^{\prime}\right)$. 
$\operatorname{Re}(7)$. The function $g$ maps $\beta$ into $\gamma$. Let $y=f^{*} \in \gamma$. If $y=1, y=g j\left(t_{0}, 0\right)$ where $j\left(t_{0}, 0\right) \in \beta$. Now assume $y \neq 1$, i.e. $f \neq i$, say

$$
\begin{aligned}
& f=\left(t_{1}, \cdots, t_{m(1)}\right) \cdots\left(t_{m-m(l)+1}, \cdots, t_{m}\right), \\
& 2 \leqq m(1) \leqq \cdots \leqq m(l), m=m(1)+\cdots+m(l) .
\end{aligned}
$$

Define $i$ and $x$ by

$$
b_{m}(i)=\left[m_{1}, \cdots, m_{l}\right]^{\#}, x=j\left(t_{m}, i\right),
$$

then $i<e_{m}, x \in \beta$ and $g(x)=y$.

We have proved that $g$ maps $\beta$ one-to-one onto $\gamma$. Note that in the proof of (7) we have suggested how to compute $g^{-1}$. It can be shown that $g$ and $g^{-1}$ have partial recursive extensions. Hence by (1) we have $\beta \simeq \gamma$ and we are done.

\section{References}

[1] J. Barback, 'Recursive functions and regressive isols', Math. Scand. 15 (1964), 29-42.

[2] J. C. E. Dekker, 'Good choice sets', Annali della Scuola Normale Superiore di Pisa, Serie III, 20 (1966), 367-393.

[3] J. C. E. Dekker, 'Infinite series of isols,' Proceedings of the Symposium on Recursive Function Theory, Amer. Math. Soc., Providence, R. I. (1962), 77-96.

[4] J. C. Dekker and J. Myhill, 'Recursive equivalence types,' Univ. California Publ. Math. (N. S.) 3(1960), 67-214.

[5] M. J. Hassett, 'Recursive equivalence types and groups', J. Symbolic Logic 34 (1969), 13-20.

[6] A. Nerode, 'Extension to isols', Ann. of Math. 73 (1961), 362-403.

[7] I. Niven and H. Zuckerman, An introduction to the theory of numbers (John Wiley and Sons, New York, 1966).

[8] W. R. Scott, Group theory (Prentice Hall, Inc., New Jersey 1964).

Bowling Green State University

Bowling Green, Ohio

U.S.A. 